3. Maan NS, Maan S, Potgieter AC, Wright IM, Belaganahalli M, Mertens PPC. Development of real-time RT-PCR assays for detection and typing of epizootic haemorrhagic disease virus. Transbound Emerg Dis. 2017;64:1120-32. https://doi.org/10.1111/tbed.12477

4. Shirafuji H, Kato T, Yamakawa M, Tanaka T, Minemori Y, Yanase T. Characterization of genome segments 2, 3 and 6 of epizootic hemorrhagic disease virus strains isolated in Japan in 1985-2013: identification of their serotypes and geographical genetic types. Infect Genet Evol. 2017;53:38-46. https://doi.org/10.1016/j.meegid.2017.05.010

5. Golender N, Bumbarov VY. Detection of epizootic hemorrhagic disease virus serotype 1, Israel. Emerg Infect Dis. 2019;25:825-7. https:/ / doi.org/10.3201/ eid2504.180149

6. Temizel EM, Yesilbag K, Batten C, Senturk S, Maan NS, Mertens PPC, et al. Epizootic hemorrhagic disease in cattle, western Turkey. Emerg Infect Dis. 2009;15:317-9. https:// doi.org/10.3201/eid1502.080572

7. Kamomae Y, Kamomae M, Ohta Y, Nabe M, Kagawa Y, Ogura Y, et al. Epizootic hemorrhagic disease virus serotype 6 infection in cattle, Japan, 2015. Emerg Infect Dis. 2018;24:902-5. https://doi.org/10.3201/eid2405.171859

8. Maan NS, Maan S, Nomikou K, Johnson DJ, El Harrak M, Madani H, et al. RT-PCR assays for seven serotypes of epizootic haemorrhagic disease virus \& their use to type strains from the Mediterranean region and North America. PLoS One. 2010;5:e12782. https://doi.org/10.1371/ journal.pone.0012782

9. Maan S, Rao S, Maan NS, Anthony SJ, Attoui H, Samuel AR, et al. Rapid cDNA synthesis and sequencing techniques for the genetic study of bluetongue and other dsRNA viruses. J Virol Methods. 2007;143:132-9. https://doi.org/10.1016/ j.jviromet.2007.02.016

10. Anthony SJ, Maan N, Maan S, Sutton G, Attoui H, Mertens PP. Genetic and phylogenetic analysis of the core proteins VP1, VP3, VP4, VP6, and VP7 of epizootic haemorrhagic disease virus (EHDV). Virus Res. 2009; 145:187-99. https://doi.org/10.1016/j.virusres.2009.07.011

Address for correspondence: Huachun Li or Heng Yang, Yunnan Animal Science and Veterinary Institute, Jindian St, Kunming 650224, China; email: Li_huachun@hotmail.com or yangheng2008.cool@163.com

\section{Pathogenic New World Relapsing Fever Borrelia in a Myotis Bat, Eastern China, 2015}

Hui-Ju Han, Jian-Wei Liu, Hong-Ling Wen, Ze-Min Li, Si-Cong Lei, Xiang-Rong Qin, Chuan-Min Zhou, Hao Yu, Xiao Xiao, Xue-Jie Yu

Author affiliations: Wuhan University, Wuhan, China (H.-J. Han, J.-W. Liu, Z.-M. Li, S.-C. Lei, X.-R. Qin, C.-M. Zhou, H. Yu, X.-J. Yu); Shandong University, Jinan, China (H.-L. Wen); Hubei University of Chinese Medicine, Wuhan (X. Xiao)

DOI: https://doi.org/10.3201/eid2612.191450

We identified Candidatus Borrelia fainii, a human pathogenic bacterium causing New World relapsing fever in a Myotis bat in eastern China. This finding expands knowledge about the geographic distribution of Borrelia spp. and the potential for infection with New World relapsing fever in China.

Rorrelia is a genus comprising 3 groups of spiro$\mathrm{B}_{\text {chetes: the Lyme disease group, the relapsing fever }}$ group, and a nonconformist third group. Typically, Lyme disease borreliae are transmitted by hard ticks and have a worldwide distribution, but most relapsing fever Borrelia are transmitted by soft ticks, except for louse-borne B. recurrentis. Relapsing fever borreliae are further classified into 2 subgroups, New World relapsing fever (NWRF) Borrelia and Old World relapsing fever, on the basis of epidemic regions and the genetic lineage of the causative agent. $B$. lonestari and B. miyamotoi are transmitted by hard ticks, but are more closely related to relapsing fever borreliae than to Lyme disease borreliae and are distributed both in the New World (North and South America and Oceania) and the Old World (Europe, Asia, and Africa). The nonconformist third group includes an orphan Borrelia species named B. turicata (1). We identified Candidatus Borrelia fainii in a Myotis bat from eastern China.

During March-October 2015, with the help of local farmers, we caught a total of 145 bats from various niches in Mengyin County, Shandong Province, China, using mist nets or butterfly nets and took tissue samples (liver, spleen, lung, or kidney). The captured bats included 4 Rhinolophus ferrumequinum and 14 Rh. pusillus from a karst cave, 26 Eptesicus serotinus from 2 farm houses, 34 Myotis fimbriatus and $10 \mathrm{M}$. ricketti from a city sewer, and $57 \mathrm{M}$. pequinius from a cave. We extracted DNA from the bat tissue, then screened for Borrelia by amplifying the $r r s, f l a B$, and $g l p Q$ genes using methods 
described previously (2-4); after gel extraction, we cloned the PCR products into pMD19 T-vectors (TaKaRa, https://www.takarabio.com) for sequencing.

We found $1 \mathrm{M}$. ricketti bat positive for Borrelia (GenBank accession nos. MG832412 for $r$ rs, MG832413 for flaB, and MG921625 for glpQ). BLAST searches showed that rrs exhibited $99.7 \%(1,491 / 1,495 \mathrm{bp})$ identity with Candidatus Borrelia fainii (accession no. LC382043), flaB exhibited 97.9\% (756/772 bp) identity with B. turicatae (accession no. CP015629), and $g l p Q$ exhibited $97.6 \%$ (859/880 bp) identity with B. parkeri (accession no. AY934633).

We performed multilocus sequence typing (MLST) by amplifying 8 housekeeping genes $(\operatorname{clp} A, \operatorname{clpX}$, nifS, pepX, pyrG, recG, rplB, and uvrA) with degenerate primers from the Borrelia MLST database (https:// pubmlst.org/borrelia). Sequence query showed that all 8 housekeeping genes were novel alleles, which were assigned the following novel allele numbers: $c l p A$ (298), clpX (261), nifS (235), pepX (264), pyrG (277), recG (292), rplB (254) and uvrA (268); the Borrelia species of this study was designated sequence type (ST) 927.

Sequences of the 8 housekeeping genes were concatenated in the order $\operatorname{clp} A, \operatorname{clpX}$, nifS, pepX, pyrG, recG, $r p l B$, and $u v r A$ and imported into MEGA7 (MEGA, https://www.megasoftware.net) for phylogenetic analysis. We constructed a phylogenetic tree using the maximum-likelihood method with the Kimura 2-parameter model. The ST927 Borrelia species was phylogenetically closely related to multiple NWRF

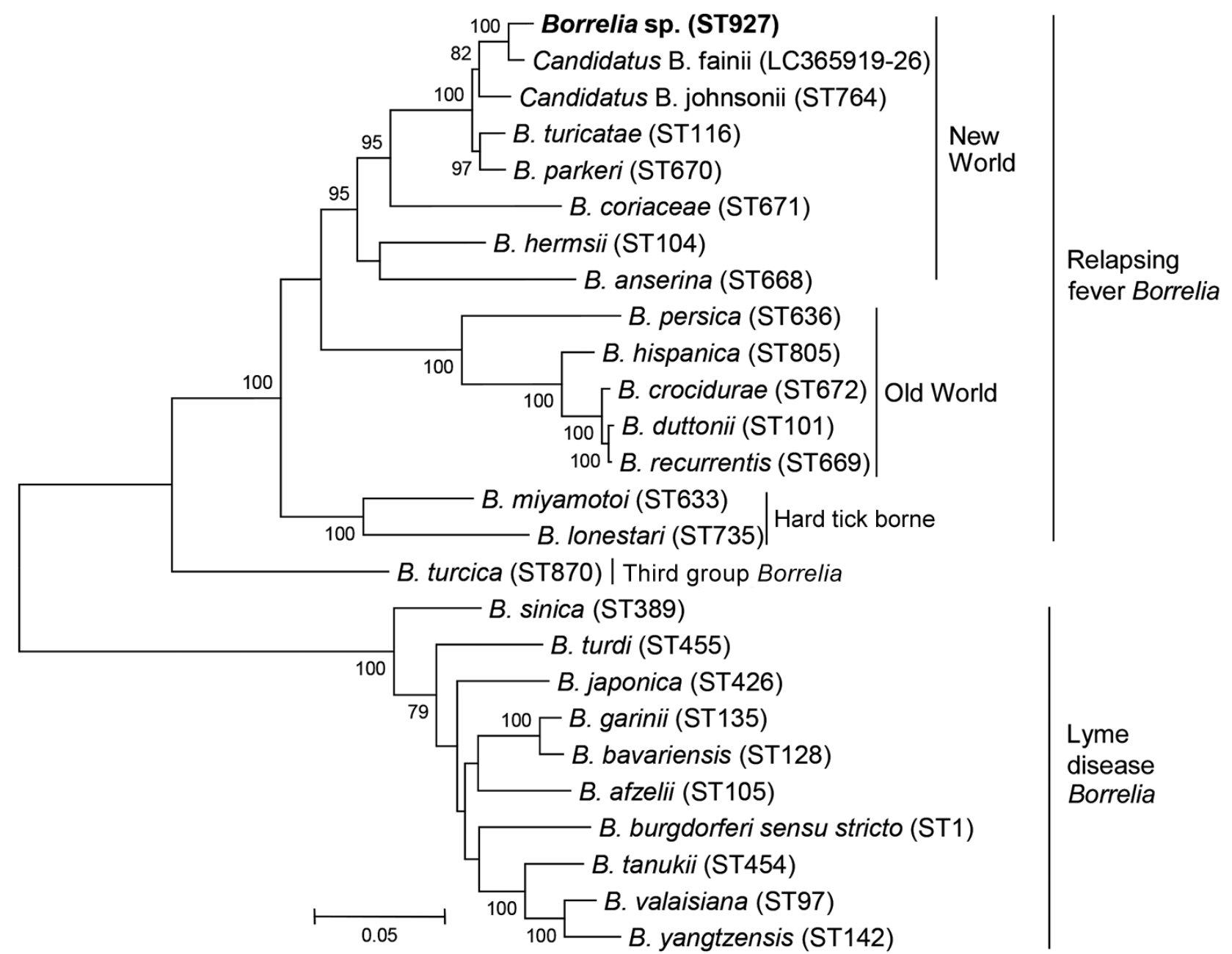

Figure. Phylogenetic analysis of Borrelia species based on 8 concatenated housekeeping genes (clpA-clpX-nifS-pepX-pyrG-recG-rplB-uvrA). Bold indicates Borrelia species identified in study of pathogenic New World relapsing fever Borrelia in a Myotis bat, eastern China, 2015. The tree was constructed by using the maximum-likelihood method in MEGA7 (https://www.megasoftware.net). Bootstrap values were calculated with 1,000 replicates. There were a total of 4,776 positions in the final dataset. Reference sequences of Borrelia species were downloaded from the Borrelia MLST database; the corresponding sequence type (ST) number of each Borrelia species is shown in parentheses. For Candidatus Borrelia fainii, the GenBank accession number is shown instead of an ST number because the 8 housekeeping gene sequences of Candidatus Borrelia fainii were only submitted to GenBank and no ST number was assigned. Scale bar indicates $5 \%$ divergence. 
Borrelia species, including B. turicatae, B. parkerii, and Candidatus Borrelia johnsonii, which are endemic in the United States, as well as Candidatus Borrelia fainii, which was recently identified in Zambia (Figure 1).

We calculated pairwise genetic distances using the Kimura 2-parameter model and identified relapsing fever Borrelia spp. using the threshold of $98.3 \%$ similarity and genetic distance 0.017 (5). Genetic distance analysis of the 8 concatenated housekeeping genes $(4,776 \mathrm{bp})$ revealed a value of 0.015 compared with Candidatus Borrelia fainii, strain Qtaro. Thus, the Borrelia sp. in our study was identified as Candidatus Borrelia fainii.

There have been several reports of NWRF Borrelia spp. in the Old World, although exclusively in Africa. A new human pathogenic Borrelia spp. was identified in Ornithodoros ticks from Tanzania that grouped together with NWRF borreliae rather than the relapsing fever-inducing spirochetes previously known to be endemic in East Africa (6). Another study described the discovery of a NWRF Borrelia, Candidatus Borrelia kalaharica, in a traveler returning from the Kalahari Desert (7). Finally, a 2019 study reported on a NWRF Borrelia-like spirochete, Candidatus Borrelia fainii, recently isolated from a febrile patient as well as from bats and bat ticks in Zambia (8).

Whether bats are reservoirs for Borrelia remains inconclusive. A new Old World relapsing fever Borrelia species, CPB1, was found responsible for the death of a Pipistrelle bat in the United Kingdom (9) and was also detected in bat soft ticks in France (10). A recent study found that bats and bat soft ticks collected from a cave in Zambia showed a high infection rate for Candidatus Borrelia fainii, and the authors proposed that bats contribute to the environmental cycle of Candidatus Borrelia fainii as hosts and bat soft ticks as vectors (8). For this study, we found only 1 bat infected with Candidatus Borrelia fainii, and it remains unclear whether bats serve as reservoirs of this Borrelia species. However, with the discovery of Candidatus Borrelia fainii in China, both health officials and physicians should pay attention to its potential emergence.

\section{Acknowledgments}

We are grateful to Dr. Gabriele Margos, the curator of the Borrelia MLST database (https:/ / pubmlst.org/borrelia), for providing us the degenerate primers for relapsing fever Borrelia MLST.

The collection of bats for microbiological studies was approved by the Ethics Committee of Prevention Medicine of Shandong University (No. 20150501).
This work was supported by the National Natural Science Funds of China (grant numbers: 81971939) and the China Postdoctoral Science Foundation Funded Project (grant numbers: 2019M662720).

\section{About the Author}

Dr. Han is a postdoctoral researcher in School of Health Sciences, Wuhan University. Her research interests include emerging infectious diseases and vector-borne diseases, especially bat-borne diseases.

\section{References}

1. Cutler SJ, Ruzic-Sabljic E, Potkonjak A. Emerging borreliaeexpanding beyond Lyme borreliosis. Mol Cell Probes. 2017;31:22-7. https://doi.org/10.1016/j.mcp.2016.08.003

2. Evans NJ, Brown JM, Demirkan I, Singh P, Getty B, Timofte D, et al. Association of unique, isolated treponemes with bovine digital dermatitis lesions. J Clin Microbiol. 2009;47:689-96. https:/ / doi.org/10.1128/JCM.01914-08

3. Assous MV, Wilamowski A, Bercovier H, Marva E. Molecular characterization of tickborne relapsing fever Borrelia, Israel. Emerg Infect Dis. 2006;12:1740-3. https://doi.org/10.3201/eid1211.060715

4. Toledo A, Anda P, Escudero R, Larsson C, Bergstrom S, Benach JL. Phylogenetic analysis of a virulent Borrelia species isolated from patients with relapsing fever. J Clin Microbiol. 2010;48:2484-9. https://doi.org/10.1128/JCM.00541-10

5. Kingry LC, Anacker M, Pritt B, Bjork J, Respicio-Kingry L, Liu G, et al. Surveillance for and discovery of Borrelia species in US patients suspected of tickborne illness. Clin Infect Dis. 2018;66:1864-71. https://doi.org/10.1093/cid/cix1107

6. Kisinza WN, McCall PJ, Mitani H, Talbert A, Fukunaga M. A newly identified tick-borne Borrelia species and relapsing fever in Tanzania. Lancet. 2003;362:1283-4. https:/ /doi.org/10.1016/S0140-6736(03)14609-0

7. Fingerle V, Pritsch M, Wächtler M, Margos G, Ruske S, Jung J, et al. "Candidatus Borrelia kalaharica" detected from a febrile traveller returning to Germany from vacation in southern Africa. PLoS Negl Trop Dis. 2016;10:e0004559. https:// doi.org/10.1371/journal.pntd.0004559

8. Qiu Y, Nakao R, Hang' ombe BM, Sato K, Kajihara M, Kanchela S, et al. Human borreliosis caused by a New World relapsing fever Borrelia-like organism in the Old World. Clin Infect Dis. 2019;69:107-12. https://doi.org/10.1093/cid/ciy850

9. Evans NJ, Bown K, Timofte D, Simpson VR, Birtles RJ. Fatal borreliosis in bat caused by relapsing fever spirochete, United Kingdom. Emerg Infect Dis. 2009;15:1331-3. https://doi.org/10.3201/eid1508.090475

10. Socolovschi C, Kernif T, Raoult D, Parola P. Borrelia, Rickettsia, and Ehrlichia species in bat ticks, France, 2010. Emerg Infect Dis. 2012;18:1966-75. https:/ / doi.org/10.3201/ eid1812.111237

Address for correspondence: Xiao Xiao, Institute of Epidemiology Research, Hubei University of Traditional Chinese Medicine, Huangjiahuxi Road No.16, Wuhan 430065, Hubei, China, email: xiaoalltheway@gmail.com; Xue-Jie Yu, State Key Laboratory of Virology, School of Health Sciences, Wuhan University, Donghu Road No. 115, Wuhan 430071, Hubei, China, email: yuxuejie@whu.edu.cn 